\title{
Interior Space Component Elements Studies of Art Deco Style (Shanghai)
}

\author{
Erde Jiang $^{1,2 a}$, Zuo Yan ${ }^{2 b}$ \\ ${ }^{1}$ ShanDong Water Polytechnic, Rizhao Shandong 276826,P.R.CHINA \\ ${ }^{2}$ Tongji University, Shanghai 200092,P.R.CHINA \\ ajiangerde18@126.com, ${ }^{\mathrm{b}}$ 813958477@qq.com
}

\section{Keywords: Art Deco,Interior Component,Furnishings}

Abstract: The thesis is to excavate and reorganize the elements of interior space form, decorative pattern, decorative materials, and space tone elements of Shanghai's Art Deco style, in order to make analysis on the space component protection and utilization value. Moreover, it improve the space component elements research of art deco style under the demands of the new age, enable the space furnishings, historical and cultural environment outlook to intermingle together, and promote the protection and utilization to interior cultural heritage of historical buildings, civilization traces, and cultural surroundings.

\section{Introduction}

The decorative Art Deco style originated from Art Nouveau (New Art Movement) and it was established in Paris' International Exposition with the theme of "decorative art of modern industry" in 1925. The Art Deco movement influenced many fields such as urban architectural design, interior design, and furnishings fields in modern China ${ }^{[1]}$. Selecting Shanghai Art Deco space as the research object, this thesis implements the case exploration with the principle of "Aims mainly to utilization with the focus on protection" so as to provide the theoretical support for the practice of feasible space renovation design.

\section{Historical relics and adaptability development}

Buildings in Art Deco style are remained in Shanghai, Harbin, Tianjin, Qingdao, Jinan, Wuhan, Ningbo, Guangzhou and other cities, among which Shanghai possesses the largest number, scatters widely, and remains the best. However, with the changes of the era and history, their decoration structures have been taken apart and transformed arbitrarily. Moreover, unreasonable functional development has enabled their interior spaces to be destroyed. Hence, the remains of furniture or decorative substances in Art Deco style used by original owners have become very little or they are not within the original space. Parts of furnishings are very difficult to be found. How to make the introduced new furnishings match with the interior space environment and inherit the interior environment of historic buildings in Art Deco style is just the starting point of this thesis.

\section{Influence factors of decorative art form}

Currently, Shanghai's buildings in Art Deco style have been protected in some degree. However, the original styles for parts of historic buildings have been damaged in some degree when conducting interior decoration. Hence, the protection and utilization to the interior spaces needs to be further improved in terms of legal constraints. Therefore, it is necessary to excavate and reorganize the space elements including interior space function layout as well as decorative elements in order to reuse the interior environment of historic buildings and inhere the historical culture. 
The interior design perception under the influence of the art deco style reflected all material and non-material aspects in that era, which influenced many cultural lives including construction, interior decoration, furniture, household appliances, etc. So far, Art Deco style, in a certain extent, is influencing the current aesthetic taste to urban space and value view. Taking advantaging of the staggered arrangement of the Art Deco geometry as well as the dynamics, twists, and turns of lines in Art Deco style conforms to the demands of the current urban nostalgia and urban retro culture. (See Fig1, 2)

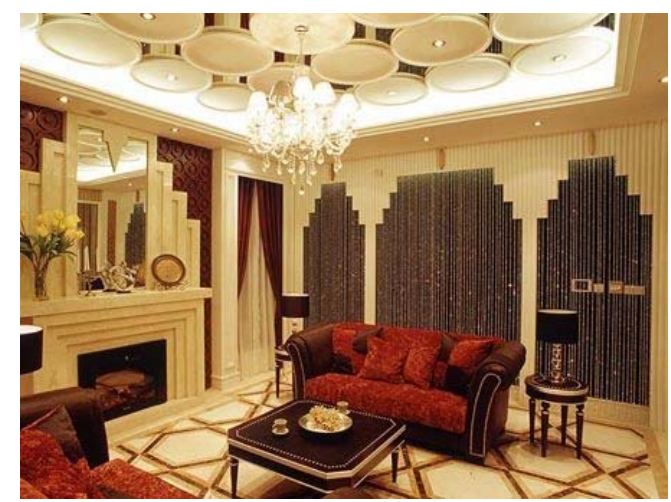

Fig 1: Habitable Room

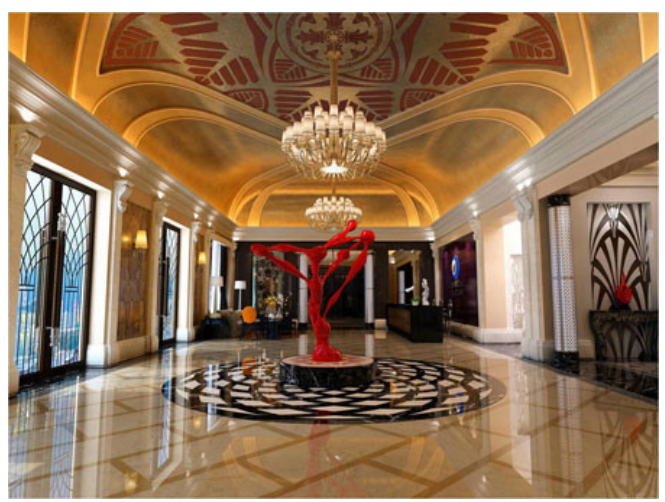

Fig 2: Hall

The space interface structure, component composition of geometry, gear or streamlined lines, and radiant sun graphics in Art Deco style manifests the beauty of machinery under the influence of industrialization. Strengthening, inhering, reusing the decorative elements including V shape, ladder shape, lightning shape, arch shape, radiation shape, circular shape, fan shape, and arc shape of the interior decorative space under the influence of Art Deco style, boosting the theory of interior Art Deco style of "reusing old things, functioning old space, and recreating the new space", straightening out the harmonious relationship between Art Deco space and humanistic spirit--absorbing, discarding, regenerating, distilling continuously and changing the unreasonable indoor space environment, and deepening the history of indoor culture including the time for human to create rich and comfortable space have become the priority in the design field.

\section{Space type analysis and method}

(1) The survey and analysis mainly aims at the type of Shanghai's representative interior space in the period of modern history, which conducts the investment and research on the style, artistic features, construction materials, construction technology, and status of engineering technology, making analysis to the value of the interior space protection and utilization. The aspects of analysis are as follows: (1) the space which reflects the historical culture and folk tradition with the era and geographical characteristics; (2) the interior historic construction style, the furniture pattern of the inner construction, and interface structure style; (3)the representative works of indoor space environment achieved by famous architect; (4) the interior space which has a special commemorative significance in the history development of revolution; (5) the representative workshop, shops, factory building, and warehouse in the history of industrial development; (6) celebrities; (7) other interior spaces of historic buildings which have special historical significances $^{[2]}$.

(2) The method of comparison is applied, which implements the comparative research on various interior construction spaces in decorative art style and sorts out the space interface and display components which is protected perfect relatively.

(3) The research adopts the method of the combination between the theory of historical heritage protection policy and on-the-spot investigation to make reasonable interior space planning and reuse. The research objects include historical \& cultural traces, indoor environment's function, furnishings items, and furniture of traditional craft \& technology, whose research fruits are applied to the actual 
room environment spaces.

\section{Aesthetic elements analysis for Interior component of the Art Deco style}

The space interface shape, volume, and structure size in Art Deco style are very important for research of decorative art. The analysis as well as relevant researches on the interior space interface paving materials, space shape pattern, space node structure approach, volume of decorative elements, form, style, and color of historical buildings contributes to the data's inductive sorting in interface organization from, article texture pattern, and furniture style pattern. It also contributes to the enlargement and perfection of interior space material in Art Deco style to merge the space furnishings into the outlook of historical \& cultural environment. (See Fig 3, Fig 4).
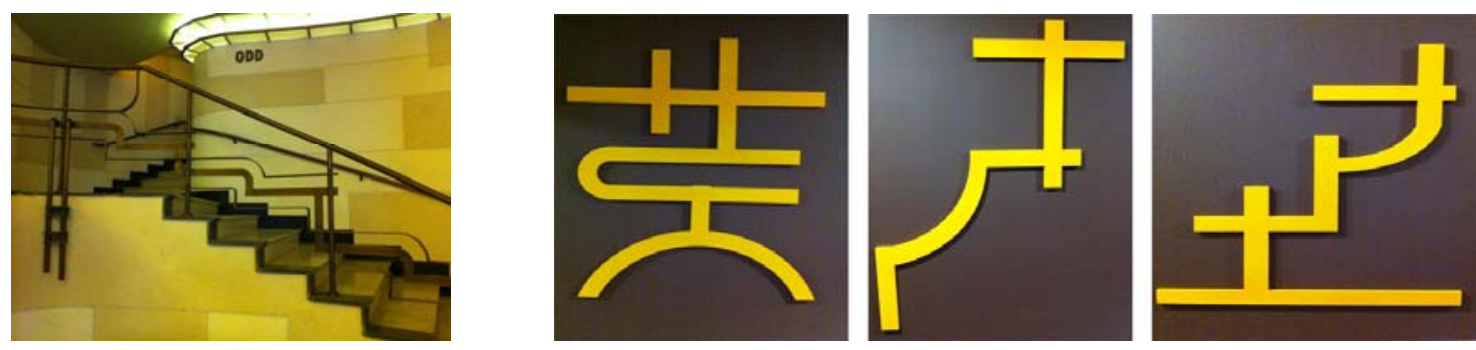

Fig 3: Stairs of Shanghai Grand Luminosity Theater

Fig 4: Decorative Symbols of Shanghai

Grand Luminosity Theater

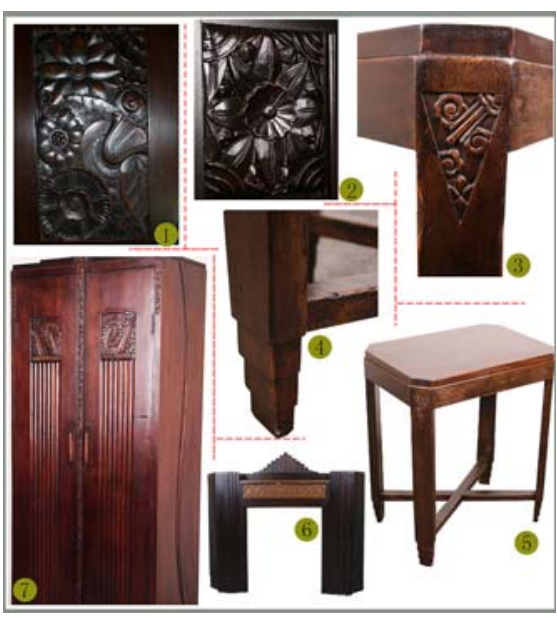

Fig 5: Decorative Art Wood Veneer

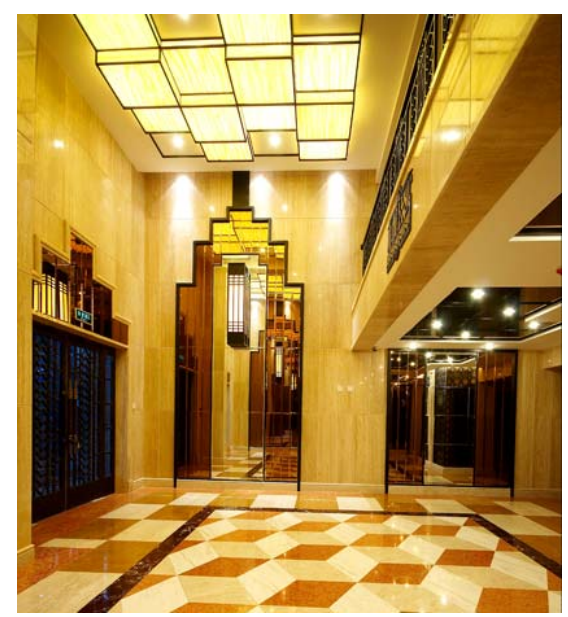

Fig 6: Hall’s Doorway Handling

(1). The interior space form and structure distributes with stepped shapes, stressing the use of vertical lines.

One feature of the interior space interface in Art Deco style is to attach great importance to embody the simplicity of the space interface and the appearance style of the furniture, emphasizes the use of vertical lines. Except that, decorative parts and materials emphasize the vertical molding. At the same time, Art Deco style focus on showing the texture of interior decoration materials, especially using the deployment of marble, granite and other hard materials with a smooth material, which can reflect the luster and the reflective effect can form a clear line reflection. (See Fig. 2)

(2).Focus on decorative geometry, radiation use, and $\mathrm{V}$ patterns

In spatial modeling, radial fan-shaped sunlight and gear-shaped or streamlined lines are used heavily, decorated with simple symmetrical geometric composition, staircase pattern, shark pattern, and zebra pattern and painted with bright and contrasting colors. The space is mainly decorated geometrically. Various simple decorative elements are utilized for interior decoration, such as 
square, circle, triangle, and diamond, forming the geometric texture and avoiding the tedious decoration pattern to achieve good decorative effect. This approach is usually used in indoor fireplace, space interface, furniture veneer and other components (See Fig. 5, 6).

(3). Use luxurious materials, decorative patterns, and psychedelic lighting to render atmosphere

Another important feature of the Art Deco is to make fine decoration with a variety of rare decoration materials, using the hanging lamp, large murals, and painted glass to pursuit the luxurious decorative effect. The color of the indoor hard material is mainly the light color, most of which use beige, light yellow and other colors. Under the irradiation of the light, it shines slightly with yellow color, reflecting the brilliant sumptuous sense ${ }^{[3]}$ (See Fig 7, Fig8).

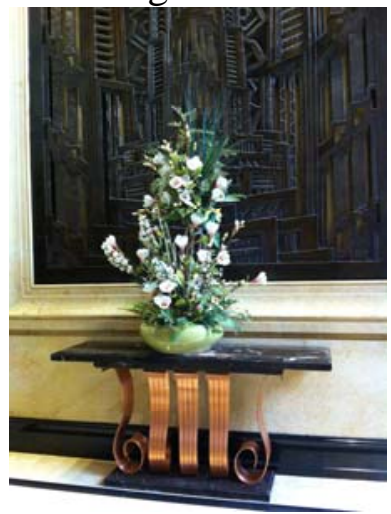

Fig 7: Relief Decorative Lamination of Jinjiang Shanghai Hall

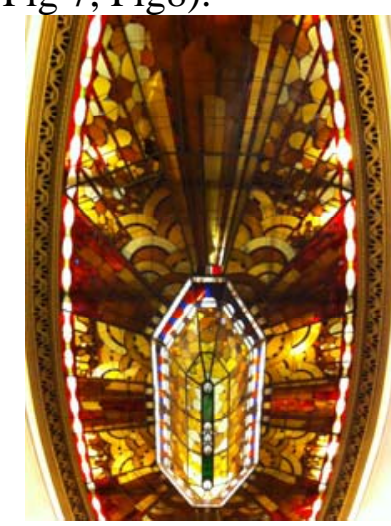

Fig 8: Flower Hall Top of Okura

Garden Hotel

Egyptian elements in Art Deco, such as fret, curve, sculpture, relief mosaic, pyramid shape, appear on the facade of the indoor walls and furniture, which expresses the noble sense pursued by the high class (See Fig5, Fig 9). In ground treatment, it makes full use of the formalist aesthetic principle such as repeated lines and symmetrical structure, which eventually give person a strong geometric sense; Floor patterns are simple segregated, forming repetitive rhythm; the line plane of carpet pattern constitutes an effective embellishment harmony and space rhythm with a certain kind of form beauty with the aim to enrich visual decorative effect. (See Fig 10)

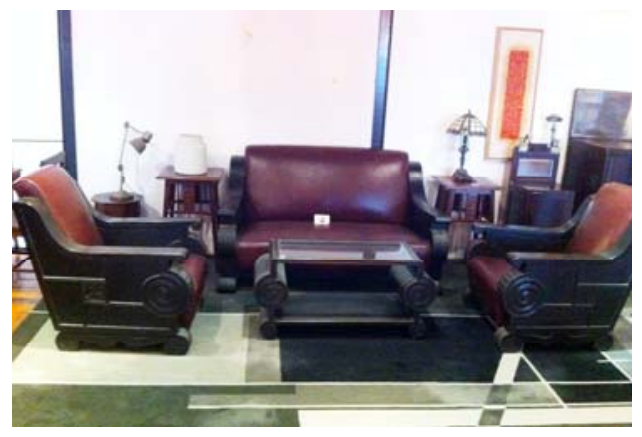

Fig 9: Space Furniture Display

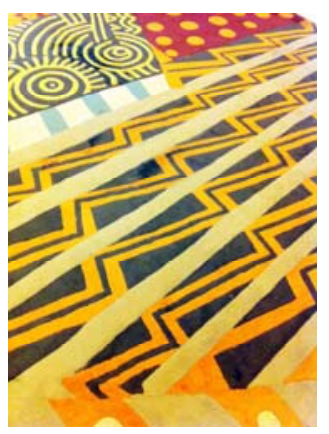

Fig 10: Carpet pattern

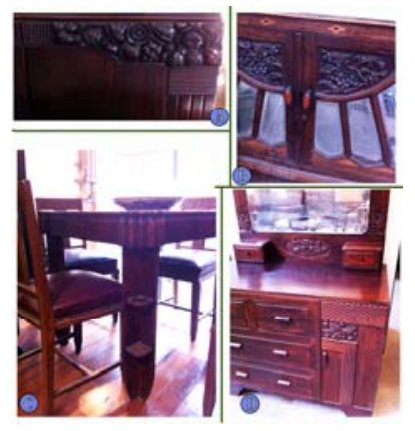

Fig11: Inlaid Relief

In the style of space interface and the doorway, the form law of symmetry, repetition, gradient is fully utilized. The stepped changes make originally simple modeling become more intriguing with rich layers. At the same time, rich-lined decoration and layer-by-layer retracted structure contour together with full-bodied and rich emotional color elements are important means to create the emotion of the decorative art. Meanwhile, the style requires that indoor lights are arranged to reflect a sense of rhythm with clear cadence and form a patterned straight line or curve ${ }^{[4]}$. (See Fig 8). 


\section{(4). Pay attention to the coordination of the decorative material tones between the overall and each part}

In the color composition, the interior decorative art style lays stress on solid color, contrast color, and classic metal color, such as warm red, melancholy blue, deep black and cold metallic color (gold, silver, bronze, etc). A variety of colors mix together, making the space full of creativity, resulting in a gorgeous visual impression. The materials of the interior space interface and furniture are carefully selected with fine workmanship, exquisite lacquer and polishing as well as plain, generous and elegant lines. Solid wood is mainly selected for the materials of decorative space and furniture, whose texture and color can produce a strong decorative characteristic through color contrast ${ }^{[5]}$. In furniture cabinet frame, reliefs are inlaid with thin wood at the edge of the cabinets. Generally, the local gold and silver are utilized to adorn moldings and turning surface so as to emphasize the furniture structure and texture as well as elegant temperament. (See Fig11)

\section{Conclusion}

Through the stage studies on interior space component elements of Art Deco Style, this thesis is to promote the protection and utilization to cultural heritage, civilization traces, and cultural surrounding of Shanghai's interior historical building, enable the space function, furnishings, and historical \& cultural environment outlook of the current interior historical buildings to intermingle together, improve the interior space design theory under the characteristics of a new era, and sum up the technical data of the "memory of the past" under the new circumstance.

\section{Acknowledgements:}

This work was financially supported by the project of domestic scholar funding for outstanding young teachers in Shandong Province.This work was also supported by National Natural Science Foundation of China (Project No.: 51278341)

\section{References:}

[1] Zuo Yan. Western Interior Design within One Hundred Year (1850-1950) [M] China architectural industry press. 2013, 26:263-264

[2] Protection Regulations for Shanghai Historical \& Cultural Area and Excellent

Historical Building [J]. Shanghai Urban Planning, 2002, 06:35-38.

[3] Guan-hong Xu . "Nostalgic and modern"___Architectural Art Deco Buildings in Shanghai [D]. Shanghai Normal University decorative building, 2010

[4] Qin min. Analysis of the interior design of the Art-deco decorative style [J]. Popular Art, 2011, 22:61

[5] Ruan Ying. Analysis on Research of Decorative Art in Shanghai Decoration Design [J]. Beauty and Era (City Edition), 2015, 01:67 